\title{
LA INVERSIÓN EN PUBLICIDAD COMO NUEVO INSTRUMENTO DE CAPITAL
}

\section{INVESTMENT IN ADVERTISING AS A NEW INSTRUMENT OF CAPITAL}

Maximiliano Gracia Hernández Profesor investigador. El Colegio del Estado de Hidalgo. Correo: graciamaximiliano@hotmailcom
Sergio Monroy Aguilar Profesor investigador.

Universidad de Quintana Roo.

\section{RESUMEN}

Resultado del proceso de globalización, la relación entre inversión y gasto en publicidad se ha profundizado en los últimos años, la competencia se incrementa por la apertura económica, haciéndose cada vez más salvaje y por ello los márgenes de ganancia se reducen; ello obliga al empresario a buscar nuevas estrategias de supervivencia y, si es posible aplicar políticas que permitan incrementar las ganancias. El documento muestra como la inversión en publicidad es una estrategia de supervivencia y un mecanismo poco viable para el incremento de los beneficios empresariales, aun cuando se percibe como una estrategia óptima.

Palabras Clave: Publicidad, Capital, Expectativas, Teoría de Juegos, Institucionalismo.

Clasificación JEL: D83

\section{ABSTRACT}

Result of the process of globalization, the relationship between investment and spending on advertising has deepened in recent years, the 
economic competition increases, becoming more wild growing openness and therefore profit margins are reduced; This obliges the employer to seek new survival strategies and if it is possible, to implement policiesto increase profits. This paper shows how investment in advertising is a survival strategy and this is not better mechanism for the increase in corporate profits, even if perceived as an optimal strategy.

Keywords: Advertising, capital, institutionalism, expectations, game theory,

Clasificación JEL: D83

\section{INTRODUCCIÓN}

Hace 200 años los economistas se preguntaban ¿qué?, ¿cómo? y ¿para quién producir? Hoy los cuestionamientos han cambiado, las preguntas clave son: ¿cuáles son las nuevas tendencias del consumo? ¿Cómo disminuir los costos e incrementar la productividad? ¿Quiénes son nuestros potenciales consumidores y como podemos llegar a ellos? Dentro de las posibles respuestas, particularmente para la última pregunta cabe introducir como elemento de análisis a la publicidad, la cual en este proceso de globalización tiene un lugar fundamental como mecanismo de generación de ganancias.

Con las nuevas tecnologías de la información vivimos un desarrollo sin precedente, se gesta un cambio estructural que intenta promover el crecimiento económico. Con este objetivo no se tiene en la escuela tradicional espacios para reconocer que las condiciones de vida social y económica han cambiado; por ello deben buscarse nuevas estructuras teóricas que lo permitan.

Consideramos en este momento que la publicidad desempeña una función fundamental que permite explicar en parte, la obtención de ganancia empresarial, pero al mismo tiempo, como se verá en el modelo, coloca a las empresas en una carrera en la que sus ganancias pueden ser mermadas, cayendo en el conocido dilema del prisionero. Se convierte así la estrategia publicitaria en una nueva arma para la competencia.

Con el proceso de globalización se profundiza la competencia económica. Para lograr subsistir en ese marco, se hace necesaria una mayor difusión de las cualidades de los productos y de la existencia de éste. El objetivo del trabajo es determinar los vínculos existentes entre economía y publicidad.

Para lograr nuestro objetivo la primera parte del documento presenta la importancia de los medios de comunicación y la publicidad en la economía, lo hace a manera de introducción general del trabajo. El segundo apartado realiza un análisis de la comunicación dentro de la historia del pensamiento económico. La tercera parte revisa de la Teoría Neoclásica al Nuevo Institucionalismo Económico. El cuarto apartado presenta la función de las expectativas en la toma de decisiones. La quinta parte realiza una serie de reflexiones acerca de la importancia de la economía de la información en el funcionamiento económico. Finalmente, el trabajo concluye con la presentación de un modelo de economía matemática, que explica la forma en que las empresas toman la decisión de: cuánto invertir en publicidad, cuando dos empresas gastan lo mismo dentro de un mismo espacio de competencia.

\section{La importancia de los medios de comunicación en la economía}

Para algunos autores como Martínez Coll (2003), la información debe ser un factor de producción, porque la tierra, el trabajo, el capital, la tecnología y know-how están en función directa de la información que acumulen; así, entre más información existe mayor posibilidad de un crecimiento económico. La información es un mecanismo que permite al empresario conocer mercados, difundir su producto, desarrollar otros 
procesos de producción, etc., los elementos antes señalados son fundamentales si se quiere vender más, los medios de comunicación serán el instrumento para lograrlo.

Se podría pensar que los medios de comunicación y la economía son dos entes aislados e independientes; sin embargo esto no es así. La economía y los medios se encuentran cada vez más vinculados; en particular la economía y la publicidad que emana de ellos.

En los últimos años se han dado pasos agigantados en el desarrollo y evolución de los medios de comunicación, ya no sólo son la televisión, la radio, los periódicos los únicos medios para difundir ideas, productos, conocimiento, etc. Hoy contamos con señal de cable desde la cual recibimos televisión de muchas partes del mundo; el internet como medio fundamental para conocer no sólo el entorno que nos rodea, sino aquel que va más allá de nuestra realidad. El desarrollo de las nuevas tecnologías de comunicación origina mayores posibilidades para llegar a los consumidores, de hecho el comercio electrónico permite ofrecer el producto y vender más. Por ende más consumo lleva a mayor producción. La teoría económica enseña que los agentes económicos son siempre tomadores de decisiones. Por tanto, una economía para ser competitiva y estar a la altura de las circunstancias actuales, necesita que todos los actores económicos se encuentren bien informados y sus expectativas sobre el futuro sean congruentes con la realidad actual y futura. Consideramos que el papel que tienen los medios de comunicación es fundamental y deberá estar basado en el compromiso con la imparcialidad, la verdad, la equidad y la ética.

Los medios de comunicación creados en el pasado, al igual que los desarrollados en este momento, ofrecen la posibilidad de llegar a cada vez más consumidores a través de mensajes publicitarios y propagandísticos. $\mathrm{Su}$ responsabilidad es asegurar audiencia y atención para que los mensajes publicitarios lleguen al mayor publico posible. Por supuesto que en la mayoría de los anuncios ofrecidos por los medios de comunicación, la venta de un producto es su principal objetivo.
Si llevamos el análisis al terreno de la empresa, considerar que el proceso de información es una actividad con elevados rendimientos a escala supongamos que una empresa a realizado un proceso de producción en el cual ya están cubiertos los costos fijos y variables, la difusión de la existencia, cualidades y virtudes de su producto dirigido a los consumidores tendrá un costo marginal muy bajo.

En los últimos años la publicidad ha pasado a formar parte fundamental y estrategia básica de supervivencia de las empresas, y es que sin publicidad es casi imposible incrementar las ganancias que coadyuvan finalmente al proceso de acumulación de capital

\section{El análisis de la comunicación dentro de la historia del pensamiento} económico.

La comunicación ha existido desde que el hombre existe, por tanto su análisis no es nuevo; lo que sí es novedoso es su tamaño, complejidad y ventajas en el mundo globalizado. Aun cuando se ha generado dentro de la ciencia económica avances importantes en la utilización del concepto de comunicación (Beltrán L. 1980), la relación entre medios de comunicación y economía no está muy clara porque se carece de un enfoque teórico que impide formalmente la vinculación de este instrumento con la ciencia económica.

A principios del siglo pasado se estudiaba la influencia de los fenómenos de masas sobre el comportamiento individual (Wolf, M. 1997); sin embargo, es a partir de los años cincuenta cuando se incrementan las posturas que consideran la inclusión de la información como parte del análisis económico (Mattelart, A. 2007; Chargoy, J. 1979). Lo anterior fue resultado de un crecimiento del sector secundario y terciario. 
Marschak, J. (1954), analiza hipotéticamente a una empresa sujeta al riesgo, el autor centra el análisis en las reglas de interacción y comunicación entre los miembros de la empresa, los cuales tienen por objeto aumentar la eficiencia de la misma. Demuestra que a mayor y mejor comunicación entre los participantes de la empresa, mayor beneficio obtiene ésta. Fritz Machlup (1962), al tratar de describirla utilizó el concepto "industria basada en el conocimiento". Posteriormente Marc Uri Porat (1977), denominó a este sector como: economía de la información. Hoy, con la tecnología digital y el Internet; la economía de la información ha crecido a grandes pasos.

Para mejorar la teoría de la inversión, Hirshleifer, J. (1965), desarrolla su análisis bajo un supuesto de futuro incierto, donde las empresas tienen distinto poder de arbitraje. Para el autor, la empresa es una unidad de decisión sujeta a riesgo e incertidumbre. El riesgo está en función del conocimiento, el cual parte de la información que se posee. Posteriormente, Hirshleifer, J. (1975) analiza y explica las acciones que realizan los agentes en los mercados especulativos, dichas actividades se encuentran en función de los riesgos y oportunidades que considera cada uno de ellos, lo cual es resultado del conocimiento. Su análisis se basa en un modelo de equilibrio general, distingue entre equilibrio informativo y no informativo.

Desde la Escuela Austriaca, es Israel Kirzner (1998), quien proporciona aportes sobre la relación entre la publicidad y la economía. El autor argumenta que en el análisis de la competencia perfecta la inclusión del análisis de la publicidad representa un avance dentro de la teoría económica. La publicidad consiste en dar información a los consumidores sobre un producto cuya existencia conocen. Parece algo improductivo; sin embargo no lo es, porque su función es persuadir al consumidor, porque aún conociendo el producto no siempre saben sus especificaciones, para ello el vendedor debe captar la atención del comprador, ayudando así a la toma de decisiones.

\section{De la teoría neoclásica al Nuevo Institucionalismo Económico.}

La teoría neoclásica supone que el individuo es racional y trabaja con información completa dentro del mercado. A través de sus decisiones, el individuo puede maximizar su bienestar porque es capaz de analizar sus restricciones y tomar la mejor solución. Lo anterior significa que bajo una perspectiva costo-beneficio, el individuo puede alcanzar sus objetivos bajo el supuesto que el mercado sea competitivo y el precio de los factores y productos no se modifique. Aquí, el papel de la publicidad consiste en proporcionar información necesaria a los agentes económicos.

Otros autores consideran que la postura de los neoclásicos, no es del todo acertada. La información en los mercados es incompleta y asimétrica. Los consumidores tienen diferente información para realizar una transacción, lo que significa que tienen información asimétrica (Akerlof 1970). Por ello, se puede afirmar que existe un mal funcionamiento natural en la economía de libre mercado; porque cada agente está interesado en mantener el dominio de la información relevante, y trata de ocultar siempre la información que puede ser usada en su contra. Además, en otras versiones de los modelos teóricos, el ser humano no es del todo racionalı, posee una racionalidad limitada a su contexto; con este argumento se explican las formas incompresibles en que funciona la bolsa de Valores; las compras compulsivas; y el marketing que de forma sutil intenta manipular a los consumidores2

Se forma así a un agente económico maximizador dentro de un escenario de información asimétrica y racionalidad limitada.

'Es conocido como el supuesto neoclásico de racionalidad ilimitada

2 Baste con observar la cantidad de productos guardados en nuestro closet, los cuales son inservibles para subsistir; realmente éstos fueron comprados como resultado de la sutileza que tiene la publicidad para
transportar a las personas hacia el consumo. Como dice Marx, el capitalismo nos lleva a la cosificación. 
La teoría neoclásica no nos permite entender íntegramente la realidad debido a la inclusión torpe de la publicidad, al enmarcarla en un entorno de incertidumbre y además al no hacer referencia explícita a que es un ámbito de competencia de las empresas que interactúan en un ambiente económico y social, porque supone explícitamente que la competencia perfecta es la ausencia completa de la competencia 3 , como puede verse claramente en cualquier manual de microeconomía neoclásica (Fuentes, R. \& Carmen M. 2001)

Frente a lo antes expuesto, el Nuevo Institucionalismo Económico presenta una postura novedosa. Considera elementos no incluidos en el análisis ortodoxo, como: los costos de transacción, la información dinámica, el accionar social e individual, fundamentalmente este último punto permite la introducción de técnicas modernas en el análisis económico, como la teoría de juegos.

\section{La función de las expectativas en la toma de decisiones}

Bajo un enfoque estrictamente económico, los individuos especulan con base en la información obtenida de los sucesos económicos por venir. Lo anterior los lleva a modificar sus acciones y su comportamiento actual. Aquí entran las expectativas, las cuales se han incorporado al análisis económico ortodoxo.

Formalmente Milton Friedman fue el primer economista que analizó las expectativas y la influencia que éstas tienen en la política económica,

3 Este resultado se puede observar claramente en que las decisiones de cuanto producir, a que precio vender, entre otras, no son tomadas por los agentes individuales que considera las acciones de los competidores; por esta razón, sus acciones son un dato más del sistema economico que determina los niveles de producción y de y no de la decisión individual o social, como se muestra en la solución de equilibrio general competitivo.

Revista de Economía - Vol. XXVIII - Núm. 78
Friedman argumentó que los individuos crean sus expectativas de forma adaptativa, ello significa que las personas generan una percepción sobre lo que va a ocurrir en el futuro, con base a la información que posee, la cual incluye lo sucedido en el pasado.

Posteriormente, surgen las expectativas racionales, su origen se observa en el análisis microeconómico neoclásicos del comportamiento de los mercados. El enfoque argumenta que las expectativas de la gente no difieren sistemáticamente de la realidad. Las expectativas se construyen utilizando toda la información disponible, que incluye una auto-modelación del comportamiento económico. Concretamente, la teoría de las expectativas racionales señala que "los participantes en el mercado no ignoran ni desechan la información y las predicciones sobre el curso futuro de la economía y sobre la actividad económica. Antes bien, anticipan racionalmente los efectos de las políticas gubernamentales y reaccionan en el presente de acuerdo con las expectativas que se han formado" (Ekelund \& Robert, 1992:585).

En este contexto, en la literatura sobre la modelización de la inclusión de la publicidad en la economía, se han usado modelos en los cuales no existe competencia perfecta 4 , de manera que se ha investigado en condiciones de mercados Monopólicos y Oligopólicos, algunos suponen (Clarke, 1976; Cable, 1972, entre otros) que la incertidumbre es la única fuerza relevante y que es factible acabarla con la publicidad; para otros (Cubbin, 1981; Friedman, 1983; Dorfman \& Steiner, 1954, entre otros)tiene que ver con la "ampliación del mercado", pero no como ámbito de competencia.

Autores como R. Dorfaman, P. Steiner (1954), J. Cubbin (1981), J. Friedman (1983), etc. Apuestan por la ampliación perpetua del mercado que intrínsecamente contradice el sustento lógico epistémico de la escasez

${ }^{4}$ Es obvia la razón, si existe competencia perfecta, entonces existe sin costo alguno, información perfecta simétrica entre los agentes economicos, los cuales conocen todo lo necesario pas s " toma de decisiones", es, etc. por lo que en estas condiciones no hay razón para contratar un servicio de publicidad. 
de los recursos. ¿Cómo se puede pensar en un mercado que tiende al infinito, cuando la mano de obra, el capital y demás factores de la producción son finitos y limitados?

Autores como P. Nelson (1970 y 1974), L. Benham (1972), G. Butters (1977), C. Shapiro (1984), Meurer y Stahl (1994) así como Muehling, Stoltman y Grossbart (1990) entre otros, han utilizado el mismo enfoque pero matizándolo con el uso de expectativas de ampliación del mercado, incluyendo ahí el marco conceptual de la incertidumbre. El enfoque de las expectativas es más moderno, pero adolece del mismo mal, suponen un mercado que puede llegar al infinito. El modelo propuesto por nosotros, no empleará incertidumbre, pero su aportación reside en que incluye a la publicidad como un arma más para la competencia entre las empresas, considerando que el mercado es finito.

\section{Competencia, medios de comunicación e información relevante}

Para autores como Coutinho (1992), las condiciones que la nueva tecnología, tanto de la información como de la producción, así como las nuevas relaciones sociales y políticas establecidas alrededor de los años 90, han promovido una aceleración de la competencia entre firmas, esta aceleración de la competencia ha tomado por asalto a los medios masivos de comunicación.

Ahora bien, esta profundización de la competencia y el reacomodo de las fuerzas políticas y económicas, tienden a reconstruir el imaginario social sobre el cual se retroalimentan y sostienen todos los hábitos de la vida social y económica de los hombres, dejando atrás el papel ético que debería jugar la comunicación masiva y transformándola en una herramienta más del poder político y económico.
Autores como Hirshleifer (1971), señalan que el exceso de información puede disminuir el bienestar. Sin embargo, para no ser presa de ello, la clave es utilizar únicamente aquella información relevante y necesaria para la adecuada toma de decisiones económicas. Por su parte, Herbet A. Simon (1985), reflexiona al respecto, argumenta que el concepto de información es cada día más complejo. Un mundo con mucha información significa el declive en la capacidad de atención por parte de los destinatarios finales. Coincidiendo con estas visiones catastrofistas de la información, Ortega y Gasset (1930) plantea que no basta con poseer información, pues es necesario poseer también las herramientas lógicas para poder procesarla.

\section{El nivel óptimo de inversión en publicidad}

Para comenzar, analizaremos el caso más puramente neoclásico en el que se compara el beneficio de invertir en publicidad, en contra del gasto en incurrir en esa inversión. Siguiendo el modelo tradicional se define una función de beneficios de la siguiente manera:

$$
\Pi=P Q(A)-c(Q)-A
$$

Donde $\Pi$ : son los beneficios de la empresa , P :Precio del bien, Q(A): Cantidad vendida del Bien, la cual depende positivamente del gasto en Publicidad, c $(\mathrm{Q})$ : Función de costo total de producción de la misma manera $Q$, representa a la cantidad producida por la empresa y A: Costo por servicios publicitarios

Definimos una estructura del gasto en publicidad la cual es equivalente a una cantidad fija sobre los bienes producidos, de la siguiente manera:

$$
A=a Q
$$


Por lo que el monto de publicidad contratado dependerá de qué tan eficiente resulta este gasto para incrementar la venta de nuestro producto. Obteniendo el resultado estándar de que se iguala el ingreso marginal por unidad de publicidad al costo marginal en publicidad que se muestra en la ecuación (3).

$$
\begin{gathered}
P \frac{d Q(A)}{d A} \frac{d A}{d Q}=\frac{d A}{d Q} \\
P a \frac{d Q(A)}{d A}=a
\end{gathered}
$$

Este modelo no puede explicar el modo en el que las empresas actúan en competencia publicitaria, por tanto, es necesario realizar algunos ajustes, con objeto de observar la forma que en un ambiente de competencia se realizan estas decisiones de las empresas; para ello utilizaremos la teoría de juegos para explicar este ambiente.

Nos encontramos inmersos en un mercado con producto diferenciado, el bien A y el bien B, los cuales son elaborados por dos empresas, la empresa 1 y la empresa 2, respectivamente; las cuales ofrecen productos que son sustitutos casi perfectos. Por ende, el objetivo de su inversión en publicidad estará determinado por convencer a los consumidores que la mercancía que se les ofrece: a) no es un sustituto perfecto, y b) el bien que ellos producen es mejor que el de la empresa rival.

Para lograrlo, deciden gastar en publicidad las cantidades de $\mathrm{A}_{1}$ y $\mathrm{A}_{2}$, el subíndice identifica a la empresa que realiza el gasto de publicidad. Como no hay incertidumbre, el mercado se reparte entre las dos empresas dependiendo de la cantidad de publi cidad que la empresa y su competidora realicen.
En estas condiciones la función de demanda inversa estará definida por (4)

$$
\begin{aligned}
& P_{1}=\alpha \frac{a_{1}}{a_{1}+a_{2}}-\beta \frac{a_{2}}{a_{1}} Q_{1}-\gamma \frac{a_{1}}{a_{2}} Q_{2} \\
& P_{2}=\alpha \frac{a_{2}}{a_{1}+a_{2}}-\beta \frac{a_{2}}{a_{1}} Q_{1}-\gamma \frac{a_{1}}{a_{2}} Q_{2}
\end{aligned}
$$

El cual representa los precios para cada uno de los bienes. Como puede observarse en el lado derecho de cada función, el precio es el mismo para cada una, y el subíndice sólo representa a cada empresa, este supuesto implica que en el modelo el precio no es ámbito de competencia.

De esta manera definimos que los espacios de competencia corresponden a la cantidad y al gasto en publicidad que cada empresa realiza, los efectos que se observan en las funciones de demanda inversa son de dos tipos:

efecto distribución del precio de reserva $\left(\alpha \frac{\mathrm{a}_{\mathrm{i}}}{\mathrm{a}_{\mathrm{i}}+\mathrm{a}_{\mathrm{j}}}\right)$, el cual se corresponde con la proporción del gasto en publicidad de la empresa con respe cto al acumulado social, y el efecto preferencia $\left(\beta \frac{\mathrm{a}_{2}}{\mathrm{a}_{1}}, \gamma \frac{\mathrm{a}_{1}}{\mathrm{a}_{2}}\right)$

que se corresponde con el factor de diferenciación del producto y la correspondiente a la competencia en gasto en publicidad. Recordemos que en nuestro modelo no hay ni tiempo ni incertidumbre, utilizando la misma nomenclatura anterior, donde:

$\Pi_{\mathrm{i}}$ : el beneficio, $\mathrm{P}_{\mathrm{i}}$ : es el precio del bien, $\mathrm{Q}_{\mathrm{i}}$ : la cantidad producida $\mathrm{y}$ vendida, $\mathrm{c}$ : el costo medio y marginal de producir $\mathrm{y} \mathrm{a}_{\mathrm{i}}$ : el costo o gasto medio y marginal de publicidad, que les corresponde $\mathrm{n}$ a la empresa $\mathrm{i}=\{1,2\}$. 


$$
\begin{aligned}
& \Pi_{1}=\mathrm{P}_{1} \mathrm{Q}_{1}-\left(\mathrm{c}+\mathrm{a}_{1}\right) \mathrm{Q}_{1} \\
& \Pi_{2}=\mathrm{P}_{2} \mathrm{Q}_{2}-\left(\mathrm{c}+\mathrm{a}_{2}\right) \mathrm{Q}_{2}
\end{aligned}
$$

Las ecuaciones (5) representan los beneficios del gasto en publicidad, donde cada empresa deseará maximizar el mismo en términos de Q y a, de manera que las funciones objetivo de cada empresa, una vez sustituidas las demandas inversas, son las siguientes:

$$
\begin{aligned}
& \operatorname{Máx}_{\mathrm{Q}_{1}, \mathrm{a}_{1}} \Pi_{1}=\left(\alpha \frac{\mathrm{a}_{1}}{\mathrm{a}_{1}+\mathrm{a}_{2}}-\beta \frac{\mathrm{a}_{2}}{\mathrm{a}_{1}} \mathrm{Q}_{1}-\gamma \frac{\mathrm{a}_{1}}{\mathrm{a}_{2}} \mathrm{Q}_{2}\right) \mathrm{Q}_{1}-\left(\mathrm{c}+\mathrm{a}_{1}\right) \mathrm{Q}_{1} \\
& \operatorname{Máx}_{\mathrm{Q}_{2}, \mathrm{a}_{2}} \Pi_{2}=\left(\alpha \frac{\mathrm{a}_{2}}{\mathrm{a}_{1}+\mathrm{a}_{2}}-\beta \frac{\mathrm{a}_{2}}{\mathrm{a}_{1}} \mathrm{Q}_{1}-\gamma \frac{\mathrm{a}_{1}}{\mathrm{a}_{2}} \mathrm{Q}_{2}\right) \mathrm{Q}_{2}-\left(\mathrm{c}+\mathrm{a}_{2}\right) \mathrm{Q}_{2}
\end{aligned}
$$

Que representan una decisión que tiene que considerar las acciones del competidor. De esta manera, las decisiones individuales óptimas tienen que responder a las acciones tomadas por cada uno de los competidores. Así, estamos dentro de la teoría de juegos.

Realizando los procesos de maximización y asumiendo que nuestros agentes son racionales y tienen conocimiento perfecto, el sistema de funciones de reacción está definido como:

$$
\begin{aligned}
& \mathrm{Q}_{1}=\frac{\mathrm{a}_{1}}{2 \beta \mathrm{a}_{2}}\left(\alpha \frac{\mathrm{a}_{1}}{\left(\mathrm{a}_{1}+\mathrm{a}_{2}\right)}-\mathrm{c}-\mathrm{a}_{1}\right)-\gamma \frac{\mathrm{a}_{1}^{2}}{2 \beta \mathrm{a}_{2}^{2}} \mathrm{Q}_{2} \\
& \mathrm{Q}_{1}=\frac{\mathrm{a}_{1}^{2}}{\beta \mathrm{a}_{2}}\left(\frac{\alpha \mathrm{a}_{1}}{\left(\mathrm{a}_{2}+\mathrm{a}_{1}\right)^{2}}+1-\frac{\alpha}{\mathrm{a}_{2}+\mathrm{a}_{1}}\right)+\frac{\mathrm{a}_{1}^{2} \gamma}{\beta \mathrm{a}_{2}^{2}} \mathrm{Q}_{2} \\
& \mathrm{Q}_{2}=\frac{\mathrm{a}_{2}}{2 \mathrm{a}_{1} \gamma}\left(\frac{\alpha \mathrm{a}_{2}}{\mathrm{a}_{1}+\mathrm{a}_{2}}-\mathrm{c}-\mathrm{a}_{2}\right)-\frac{\mathrm{a}_{2}^{2} \beta}{2 \mathrm{a}_{1}^{2} \gamma} \mathrm{Q}_{1} \\
& \mathrm{Q}_{2}=\frac{\mathrm{a}_{2}^{2}}{\gamma \mathrm{a}_{1}}\left(\frac{\alpha \mathrm{a}_{2}}{\left(\mathrm{a}_{2}+\mathrm{a}_{1}\right)^{2}}+1-\frac{\alpha}{\mathrm{a}_{2}+\mathrm{a}_{1}}\right)+\frac{\mathrm{a}_{2}^{2} \beta}{\gamma \mathrm{a}_{1}^{2}} \mathrm{Q}_{1}
\end{aligned}
$$

Donde cada $Q_{i}$ representa una curva de reacción de la empresa iesima, la primera es para cantidades y la segunda para el gasto en publicidad.

Así, la cantidad a producir que tiene cada una de las empresas para responder mejor a los gastos de publicidad y producción realizados por la otra empresa, está representada por el sistema de ecuaciones(7) de manera que podemos ver en ella los límites y la estrategia del juego.

Ahora analicemos con cuidado, en las funciones (7), tenemos un sistema casi lineal en términos de $Q_{1}$ y $Q_{2}$ pero no lineal en términos de ${ }^{a_{1}}$ y $a_{2}$ de manera que las soluciones integrales del sistema son muy complicados para obtener solución analítica y más cuando no tenemos los valores de los parámetros del sistema. Así que hemos decidido hacer un proceso de simplificación, para ello sustituiremos la primera ecuación del sistema (7) en la segunda ecuación del mismo sistema(7) y viceversa. La razón de este esquema es que estas ecuaciones son las que nos permiten tener en consideración el criterio de maximización de los beneficios, tomando en cuenta las decisiones de los mismos en términos de su producto. Que es su variable de decisión.

Con ello, el nuevo sistema en términos de cantidades de producto quedará representado como:

$$
\begin{aligned}
& Q_{1}=-\frac{\left.\left(a_{1} a_{2}-2 a_{1}^{2}\right) \alpha+\left(a_{1} a_{2}+a_{1}^{2}\right) c-a_{1} a_{2}^{2}+a_{1}^{2} a_{2}+2 a_{1}^{3}\right)}{\left(3 a_{2}^{2}+3 a_{1} a_{2}\right) \beta} \\
& Q_{2}=\frac{\left.\left(2 a_{2}^{2}-a_{1} a_{2}\right) \alpha+\left(-a_{2}^{2}-a_{1} a_{2}\right) c-2 a_{2}^{3}-a_{1} a_{2}^{2}+a_{1}^{2} a_{2}\right)}{\left(3 a_{1} a_{2}+3 a_{1}^{2}\right) \gamma}
\end{aligned}
$$


Así el subsistema de cantidades quedará expresado en términos de la competencia en gastos de publicidad. Que en adelante será la variable de toma de decisiones. Pero como el sistema, sigue siendo no lineal, utilizaremos el análisis matemático para encontrar sus implicaciones en la toma de decisiones de nuestros empresarios representativos.

Para ello, sabemos que nuestros empresarios conocen el subsistema de cantidades, de manera que toma su decisión considerando que el competidos ha tomado una decisión al respecto, lo que implica que conoce cual será su demanda residual y ante ella toma su decisión de invertir en publicidad porque sabe cuanto obtendrá por ello.

De esta manera, cuando el gasto en publicidad de la empresa tiende a cero, suponiendo que la otra hace una inversión en publicidad positiva, el valor de sus ventas es cero

$$
\lim _{a_{1} \rightarrow o}\left(Q_{1}=-\frac{\left.\left(a_{1} a_{2}-2 a_{1}^{2}\right) \alpha+\left(a_{1} a_{2}+a_{1}^{2}\right) c-a_{1} a_{2}^{2}+a_{1}^{2} a_{2}+2 a_{1}^{3}\right)}{\left(3 a_{2}^{2}+3 a_{1} a_{2}\right) \beta}\right)=0
$$

Debido a que $\mathrm{a}_{1}\left(2 \alpha-\mathrm{c}-2 \mathrm{a}_{1}\right) \beta>>0 \quad$ representa que los gastos o costos asumidos por la empresa y ponderados por las preferencias de los consumidores, no son inferiores al costo de reserva. Con esto garantizamos que el costo de reserva y por consiguiente la demanda, permita la consecución de ganancias y excluyendo el caso de que la demanda no sea de tal magnitud que la empresa obtenga recurrentemente perdidas.

Este resultado implica que si no gasta nada en publicidad y su contraparte sí, no venderá nada de su producto, por ello se verá motivado a gastar en publicidad. Ahora bien, supongamos que gasta todo su ingreso de operación marginal, en publicidad. Lo que implica que la empresa decide gastar todo el diferencial del precio menos los costos de producción en publicidad. Siguiendo la lógica de maximización de beneficios; entonces, supo- niendo que la otra empresa gasta menos lo que implicaría que:

$\mathrm{a}_{1} \mathrm{Q}_{1}>\mathrm{a}_{2} \mathrm{Q}_{2}$; dado que la empresa 1 conoce cuanto gastará en publicidad la empresa 2, él decidirá gastar todo el diferencial entre precio y sus costos de operación, para quedarse con un mayor segmento del mercado y dar una señal a su competidor de que esta dispuesto a sacrificar utilidades con tal de quedarse en el mercado; con esta estrategia, puede observarse que la cantidad producida y vendida es positiva y como se observará mas adelante, es superior a la cantidad cuando ambas empresas gastan lo mismo en publicidad.

$$
\begin{aligned}
& \lim _{a_{1} \rightarrow P_{1}-c}\left(Q_{1}=-\frac{\left.\left(a_{1} a_{2}-2 a_{1}^{2}\right) \alpha+\left(a_{1} a_{2}+a_{1}^{2}\right) c-a_{1} a_{2}^{2}+a_{1}^{2} a_{2}+2 a_{1}^{3}\right)}{\left(3 a_{2}^{2}+3 a_{1} a_{2}\right) \beta}\right) \\
& \lim _{a_{1} \rightarrow P_{1}-c}\left(Q_{1}\right)=-\frac{2 P_{1}^{3}+\left(-2 \alpha-5 c+a_{2}\right) P_{1}^{2}+\left(\left(4 c+a_{2}\right) \alpha+4 c^{2}-a_{2} c-a_{2}^{2}\right) P_{1}+\left(-2 c^{2}-a_{2} c\right) \alpha-c^{3}+a_{2}^{2} c}{3 a_{2} \beta P_{1}+\left(3 a_{2}^{2}-3 a_{2} c\right) \beta}
\end{aligned}
$$

Continuando con nuestra investigación, para ver lo que sucede con la empresa 1 con las decisiones de la empresa 2, haremos el mismo análisis, en primer lugar veremos que ocurre cuando no decide gastar en publicidad la empresa 2 , lo que nos muestra que el mercado completo es acaparado por la empresa 1:

$\lim _{a_{2} \rightarrow o}\left(Q_{1}=-\frac{\left.\left(a_{1} a_{2}-2 a_{1}^{2}\right) \alpha+\left(a_{1} a_{2}+a_{1}^{2}\right) c-a_{1} a_{2}^{2}+a_{1}^{2} a_{2}+2 a_{1}^{3}\right)}{\left(3 a_{2}^{2}+3 a_{1} a_{2}\right) \beta}\right)=\infty$

Por la misma razón de antes $\mathrm{a}_{1}\left(2 \alpha-\mathrm{c}-2 \mathrm{a}_{1}\right) \beta>>0$; esta inecuación representa la garantía de que la demanda es capas de absorber el producto de la empresa y garantizar la ganancia no negativa. 
De la misma manera si la empresa 2 decide gastar todo su beneficio marginal en publicidad, es de esperar que la empresa 1 tenga el siguiente comportamiento.

$$
\begin{gathered}
\lim _{a_{2} \rightarrow P_{2}-c}\left(Q_{1}=-\frac{\left.\left(a_{1} a_{2}-2 a_{1}^{2}\right) \alpha+\left(a_{1} a_{2}+a_{1}^{2}\right) c-a_{1} a_{2}^{2}+a_{1}^{2} a_{2}+2 a_{1}^{3}\right)}{\left(3 a_{2}^{2}+3 a_{1} a_{2}\right) \beta}\right) \\
\lim _{a_{2} \rightarrow P_{2}-c}\left(Q_{1}\right)=\frac{\left.a_{1} P_{2}^{2}+\left(-a_{1} \alpha-3 a_{1} c-a_{1}^{2}\right) P_{2}+\left(a_{1} c+2 a_{1}^{2}\right) \alpha+2 a_{1} c^{2}-2 a_{1}^{3}\right)}{3 \beta P_{2}^{2}+\left(3 a_{1}-6 c\right) \beta P_{2}+\left(3 c^{2}-3 a_{1} c\right) \beta}
\end{gathered}
$$

Lo que puede verse que es menor la producción vendida, porque le corresponde menor poder de mercado, que le otorga el gasto en publicidad. Por lo que nos falta analizar el último caso, no sin antes recalcar que lo mismo es cierto para la empresa dos, por el simple hecho que son "simétricas" al poseer la misma tecnología y acceso a tecnología y publicidad, entre ellas no hay diferencia real.

Finalmente, si el gasto en publicidad de las dos empresas coincide de $a_{1}=a_{2}$

manera que

, la producción de la empresa 1 será:

$$
\mathrm{Q}_{1}=\frac{\alpha-2 \mathrm{c}-2 \mathrm{a}_{1}}{6 \beta}
$$

Por lo que podemos concluir lo siguiente, en relación a la empresa 1, su gasto en publicidad tiene un efecto positivo en su nivel de ventas 5 (salvo el caso en que sean iguales), el gasto de los competidores tendrá un impacto negativo, lo cual lo obliga a tener un gasto creciente en publicidad.

5 Veamos con detenimiento la ecuación (13), la cual corresponde al caso en que ambas empresas gastan lo mismo en publicidad, en este caso y sólo en este caso, implica que se reparten el mercado en partes iguales, por lo que el gasto en publicidad es un costo adicional al costo marginal de producir, por ello su impacto es determinar fácilmente que el gasto de publicidad de la empresa uno es positivo en cantidad. Lo que implicaedue la empresa con mayor gasto relativo poseerá una proporción mayor y de la misma magnitud del mercado. Es decir, si decidiera gastar más que su adversario y logra que su porcentaje de gasto en publicidad sea mayor, anará esa misma proporción mayor de mercado.

Revista de Economía - Vol. XXVIII - Núm. 78
De manera que el monto máximo de gasto en publicidad será igual al ingreso marginal de operación. Lo propio es válido con la empresa 2, por razones expuestas arriba.

Ahora bien, para mantener el esquema, es necesario obtener $\quad Q_{2}$ bajo el caso de que ${ }^{a_{2}=a_{1}}$ y ver qué pasa en la distribución del mercado.

Para ello calculamos:

$$
\mathrm{Q}_{2}=\frac{\alpha-2 \mathrm{c}-2 \mathrm{a}_{2}}{6 \gamma}
$$

Dividiendo ¡Error! No se encuentra el origen de la referencia. entre (1) $a_{1}=a_{2}$

, y como

tenem os:

$$
\frac{Q_{1}}{Q_{2}}=\frac{\frac{\alpha-2 \mathrm{c}-2 \mathrm{a}_{2}}{6 \beta}}{\frac{\alpha-2 \mathrm{c}-2 \mathrm{a}_{2}}{6 \gamma}}=\frac{\lambda}{\beta}
$$

De manera que solo serán iguales las ventas en caso de que el producto de ambas empresas sea intrínsecamente el mismo, lo cual implica que se repartirán el mercado según sea el grado de diferenciación real entre los productos.

Para ver más claramente los dos posibles equilibrios, es importante ver el beneficio obtenido por cada uno de los productores. Así evaluaremos primero el caso en el cual, ambas empresas se reparten el mercado con un gasto en publicidad igual a cero. Como el gasto en publicidad en las dos es cero, entonces el precio de cada empresa será

$$
P_{1}=P_{2}=\frac{2}{3}(\alpha-c)
$$


Por lo que su beneficio será

$$
\Pi_{1}=\frac{\alpha^{2}-\mathrm{c} \alpha-9 \mathrm{c} \beta+2 \mathrm{c}^{2}}{9 \beta}
$$

El otro equilibrio es, cuando $a_{1}=a_{2}=P-c$ en este caso no es

necesario suponer que el producto es el mismo, pues

determinan el precio del mercado, el cual es:

$$
P_{1}=P_{2}=P=\frac{\alpha+4 c}{6}
$$

Por lo que los beneficios serán: $\quad \Pi_{1}=0$

De aquí se desprende una conclusión interesantísima, de los dos equilibrios posibles, de dos empresas en las que se produce con la misma tecnología y ambas tienen el mismo gasto de publicidad porque tienen el mismo precio de mercado, entonces el beneficio de no gastar en publicidad, es mayor al beneficio de gastar en la misma; de manera que la competencia en publicidad, bajo las condiciones descritas, supone la existencia de un dilema del prisionero en el que a las empresas les convendría coludirse para obtener una ganancia positiva pero no lo hacen porque los incentivos a traicionar son mayores y por lo tanto gastan en publicidad, esperando obtener esas ganancias mayores y al final no obtienen ninguna.

\section{CONCLUSIONES}

El desarrollo de los medios de comunicación permite a los individuos, tanto consumidores como productores, tener diferentes opciones para la la toma de las mejores decisiones, las cuales se deben abordar bajo diferentes escenarios, muchas de ellas con expectativas positivas o negativas, pero finalmente la deliberación que se considere tendrá efectos en el funcionamiento económico.

Los medios de comunicación, y en particular la publicidad que de ellos emana, forman parte de los costos de quien los utiliza, los cuales se han incrementado como resultado del aumento en las innovaciones tecnológicas y de una mayor competencia.

En el modelo presentado se concluye que dadas dos empresas, la inversión en publicidad tiene efectos positivos en su nivel de ventas únicamente en el caso de que se tenga una estrategia mucho más agresiva que los rivales. Lo cual obliga al competidor a generar un gasto creciente en publicidad. De manera que el monto máximo de gasto en publicidad será igual al ingreso marginal de operación.

Si suponemos dos equilibrios posibles, con dos empresas en las que se produce con la misma tecnología y ambas tienen el mismo gasto de publicidad, porque tienen el mismo precio de mercado; entonces el beneficio de no gastar en publicidad, es mayor al beneficio de gastar en la misma; de manera tal, que la competencia en publicidad bajo las condiciones descritas anteriormente, supone la existencia de un dilema del prisionero, en el que a las empresas les conviene coludirse para obtener una ganancia positiva, pero no lo hacen porque los incentivos a traicionar son mayores y por lo tanto gastan en publicidad con objetivo de obtener ganancias mayores, sin embargo, al final no las obtienen.

Este resultado indica que es éticamente incorrecto dejar que los empresarios en libertad compitan con estrategias publicitarias cada vez más agresivas, porque ellas disminuyen el bienestar social por las dos vías, disminuyen los excedentes de los productores, pero fundamentalmente el de los consumidores, que ahora son presa de los espíritus que desean mantenerse en el mercado. 


\section{REFERENCIAS}

Akerlof, G. (1970). “The market for lemons. Quality Uncertainty and the market mechanism", Quarterly Journal of Economics, 84 (3), Harvard University's Department of Economics, Cambridge, MA, 481-500 pp.

Beltrán, L (1980). "Farewell to aristotle horizontal communication" Revista Communication, volumen 5, número I, New York, London and Paris, Edit. Karl Erik Rosengren. 1-7 pp.

Benham, L. (1972). "The effect of advertising on the price of eye-glasses", Journal of Law and Economics, 15, 337-52.

Butters, G. (1970). "Equilibrium distribution of sales and advertising prices", Review of Economic Studies, 44, 465-91.

Cable, J. (1972). "Market Structure, Advertising Policy, and Inter-Market Differences in Advertising Intensity," en K. Cowling, eds, Market Structure and Corporate Behavior; Theory and Empirical Analysis of the Firm.

Clarke, D.G (1976). "Econometric measurement of the duration of advertising effect on sales", Journal of Marketing Research, 13 (4), 345-57

Cubbin, J. S. (1981). "Advertising and the theory of entry barriers", Económica, 48 (Agosto), 289-98

Coutinho, L. (1992). "A terceira revoluçao industrial e tecnológica: As grandes tendências de mudanças". Economia e Sociedade. 1 (8), Universidade Estadual de Campinas, Campiñas, Brasil; pp. 69-87.

Chargoy, J. (1979). Sistemas de información y análisis económico, UNAM, México, $261 \mathrm{pp}$

Dorfman, R. y Steiner, P. O. (1954). "Optimal advertising and optimal quality", American Economic Review, 44 (5), 826-36.

Ekelund, R. \&, Robert, F. (2004). Historia de la teoría económica y su método, $3^{\mathrm{a}}$ ed. Mc Graw Hill, Madrid. $731 \mathrm{pp}$.

Friedman, J. W. (1983). "Advertising and oligopolistic equilibrium", Bell Journal of Economics, 14 (2), 46-73
Fuentes, R. \& Carmen M. (2001). Introducción a la economía, edit Club Universitario, Madrid, $277 \mathrm{pp}$.

Herbert, S. (1985). "Human nature in politics: The Dialogue of Psychology with Political Science", American Political Science Review 79 (2), New Hampshire, Washington, DC, 293-304 pp.

Hirshleifer, J. (1975). "Speculation and equilibrium: Information, risk, and markets". Quarterly Journal of Economics 89 (4), Harvard University's Department of Economics, Cambridge, MA, 520-542 pp.

Hirshleifer, J. (1965). "Investment decision under uncertainty: Choicetheoretic approaches", The Quarterly Journal of Economics 79 (4), Harvard University's Department of Econ., Cambridge, MA, 509-536 pp.

Kirzner, I. (1998). Competencia y empresarialidad, Unión Editorial Madrid, $304 \mathrm{pp}$.

Machlup, F. (1962). The production and distribution of knowledge in the United States. Princeton University Press, Princeton New Jersey, $420 \mathrm{pp}$.

Marschak, J. (1954). Towards an economic theory of organization and information, en Thrall et al., editores, Decision Processes. Disponible en cowles.econ.yale.edu/P/cp/p00b/p0095.pdf.Consult. el 2/12 2008

Martin, S. (1998). Advanced industrial economics. Blacwell, Oxford England. $659 \mathrm{pp}$

Martínez C. (2003). El ser humano, la información y la economía. Contribuciones a la Economía. Disponible en: www.eumed.net/ce/jcmc-inf.htm. Consultado el 17/11/2008. Consultado el 2/2/2009

Mattelart, A. (2007). Historia de la sociedad de la información, edit. Paidos, Barcelona, 209 pp.

Meurer, M y Stahl, D. (1994). "Informative advertising and product match", International Journal o Industrial Organization, 12, 1-9

Muehling, D. Stoltman, J. y Grossbart, S. (1990). "The impact of compara tive advertising on level of message involvement", Journal of Advertising, 19, 41-50. 
Nelson, P. (1970). "Information and consumer behavior", Journal of Political Economy 78 (2), University of Chicago, 311-329 pp.

Nelson, P. (1974). "Advertising as information", Journal of Political Economy 82 (4), University of Chicago, Chicago, 729-754 pp.

Ortega y Gasset, J. (1930). La rebelión de las masas, Editorial Porrúa, México D.F., 214pp

Shappiro, C. (1980). "Advertising and welfare: Comment", Bell Journa of Economics, 11, 749-52.

Shy, Oz. (1998). Industrial organization. Theory and applications. MIT Press, Cambridge, Massachusetts, $466 \mathrm{pp}$

Wolf, M. (1997). La investigación de la comunicación de masas: crítica y perspectivas, trad. Carmen Artal, Edit. Paidos, México, 318 pp. 